\title{
Proton Magnetic Resonance Spectroscopy of the Central Nervous System
}

\author{
Evanthia Kousi, loannis Tsougos and Kapsalaki Eftychia
}

Additional information is available at the end of the chapter

http://dx.doi.org/10.5772/53892

\section{Introduction}

Early and accurate diagnosis of patients with cerebral demyelinating or infection diseases, space occupying mass lesions and neurological deficits, is essential for optimum treatment decision concerning the administration of specific medication or chemotherapeutic agents, radiation therapy and/or surgical resection.

Currently, conventional MR imaging (MRI) is considered to be an established and useful tool in brain disease detection and it is widely chosen as the initial examination step in patients suspected of brain lesions as it is effective in simultaneously characterizing the soft tissue, cerebrospinal fluid (CSF) spaces, and blood vessels. It is a flexible imaging modality for which contrast can be extensively manipulated without patient burdening by ionizing radiation. Nevertheless, the accurate characterization of brain lesions with MR imaging remains problematic in several cases as the sensitivity and specificity with which this modality defines several brain lesions remains limited [1].

To overcome the aforementioned limitation, the development of new imaging techniques is required, in order to highlight functional or metabolic properties of brain tissue. Proton Magnetic resonance spectroscopy ( $\left.{ }^{1} \mathrm{H}-\mathrm{MRS}\right)$ is one such technique which provides a noninvasive method for characterizing the cellular biochemistry which underlies brain pathologies, as well as for monitoring the biochemical changes after treatment in vivo. It is considered as a bridge between metabolism and the anatomic and physiological studies available from MRI [2].

Until now, ${ }^{1} \mathrm{H}-\mathrm{MRS}$ has been used as both a research and a clinical tool for detecting abnormalities -visible or not yet visible- on conventional MRI. Suggestively, MollerHartman et al. reported that when only the MR images used for radiological diagnosis of focal intracranial mass lesions, their type and grade were correctly identified in $55 \%$ of the 
cases, however, the addition of MR spectroscopic information significantly raised the proportion of correctly diagnosed cases to $71 \%$ [3].

${ }^{1} \mathrm{H}$-MRS has been always challenging in terms of its technical requisites (field strength, gradients, coils and software), as well as the accurate metabolic interpretation with regards to pathologic processes. However, the clinical applications of ${ }^{1} \mathrm{H}-\mathrm{MRS}$ are continuously increasing as the clinical hardware have become more robust and user-friendly along with improved data analysis, spectra post-processing techniques and metabolite interpretation confidence.

The purpose of this chapter is to provide a thorough review concerning the current status of ${ }^{1} \mathrm{H}-\mathrm{MRS}$ in terms of its clinical usefulness as well as its technical prerequisites.

\section{Basic principles}

In order to introduce the basic concepts and terminology of ${ }^{1} \mathrm{H}-\mathrm{MRS}$, the basic principles of MRS are briefly described below.

Proton is a charged particle with spin, and exhibits the electromagnetic properties of a dipole magnet. When protons are placed in an external magnetic field $B_{0}$, they align themselves along the direction of the field (either parallel or anti-parallel) and demonstrate a circular oscillation. The frequency of this circular motion (called Larmor frequency) is dependent on the strength of the local magnetic field and the molecular structures at which protons belong. This can be expressed by the Larmor equation:

$$
\omega_{0}=\gamma \mathbf{B}_{0}
$$

where $\omega_{0}$ is the Larmor frequency, $\gamma$ is the gyromagnetic ratio specific for the nuclei, and $\mathbf{B}_{0}$ is the strength of the external magnetic field.

When electromagnetic energy (in the form of a RF pulse) is supplied at this frequency, the molecules absorb this energy and change their alignment. When the RF pulse is switched off, the molecules realign themselves to the magnetic field by releasing their absorbed energy. This released energy is the basis of the MR signal [4].

${ }^{1} \mathrm{H}$-MRS uses the same hardware as conventional MRI, however, their main difference is that the frequency of the MR signal is used to encode different types of information. MRI generates structural images, whereas ${ }^{1} \mathrm{H}$-MRS provides chemical information about the tissue under study.

Although recent studies have shown promise for the use of ${ }^{1} \mathrm{H}-\mathrm{MRS}$ to investigate malignant processes to prostate [5], breast [6], skeletal muscles [7], cervical and ovarian cancer [8], the overwhelming number of applications have been demonstrated in the brain, due to the absence of free lipid signals in normal cerebrum, relative ease of shimming, and lack of inherent motion artifacts.

The output of ${ }^{1} \mathrm{H}-\mathrm{MRS}$ is a spectrum which is described by two axes as it is illustrated in figure 1 . The vertical axis (y) represents the signal intensity or relative concentration for the 
various cerebral metabolites and the horizontal axis $(\mathrm{x})$ serves to describe the frequency chemical shift in parts per million (ppm). The nature of the chemical shift effect is to produce a change in the resonant frequency for nuclei of the same type attached to different chemical species. It is due to variations in surrounding electron clouds of neighboring atoms, which shield nuclei from the main magnetic field $\left(\mathrm{B}_{0}\right)$. The resulting frequency difference can be used to identify the presence of important chemical compounds. Within the spectrum, metabolites are characterized by one or more peaks with a certain resonance frequency, line width (full width at half maximum of the peak's height, FWHM), line shape (e.g., lorentzian or Gaussian), phase, and peak area according to the number of protons that contribute to the observed signal. By monitoring those peak factors, ${ }^{1} \mathrm{H}-\mathrm{MRS}$ can provide a qualitative and/or a quantitative analysis of a number of metabolites within the brain if a reference of known metabolite concentration is used at a particular field strength [9].

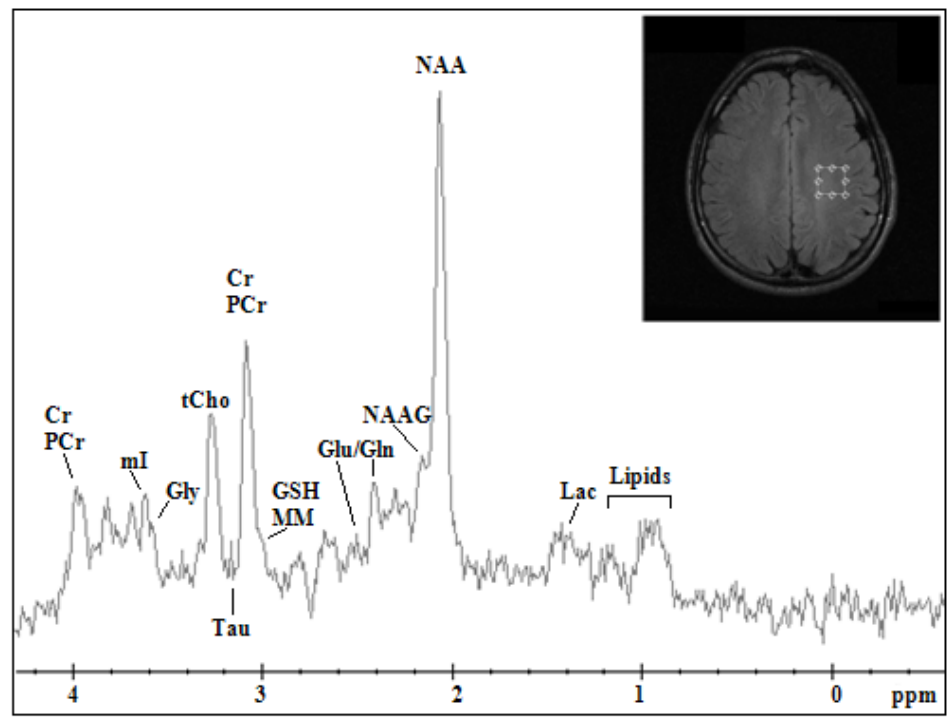

Figure 1. Proton MR spectrum from Parietal White Metter measured at $3 \mathrm{~T}$ in the normal human brain of a 19-year-old volunteer.

\section{Neurospectroscopy biochemical features and their clinical significance}

Accurate classification of cerebral lesions by in-vivo ${ }^{1} \mathrm{H}-\mathrm{MRS}$ requires determination of the relationship between metabolic profile and pathologic processes.

The assignment and clinical significance of the basic resonances in a spectrum as well as the less commonly detected compounds are discussed below:

$\mathrm{N}$-Acetyl Aspartate (NAA) in ${ }^{1} \mathrm{H}-\mathrm{MR}$ spectra of normal cerebral tissue, is the most prominent resonance which originates from the methyl group of NAA at 2.01ppm with a contribution from neurotransmitter $\mathrm{N}$-aspartyl-glutamate (NAAG) (figure 1). NAA is exclusively 
localized in central and peripheral nervous system and it is synthesized in brain mitochondria. Its concentration subtly varies in different parts of the brain [10] and undergoes large developmental changes, increasing from $4.82 \mathrm{mM}$ at birth to $8.89 \mathrm{mM}$ in adulthood. Although NAA is considered as a neuronal marker and equate with neuronal density and viability, its exact function remains largely unknown.

The utility of NAA, as an axonal marker is supported by the loss of NAA in many white matter diseases, including leukodystrophies [11], multiple sclerosis (MS) [12] and hypoxic encephalopathy [13], chronic stages of stoke [14] and tumors [1, 2, 9]. However, there are cases when the abnormal levels of NAA do not reflect changes in neuronal density, but rather a perturbation of the synthetic and degradation pathways of NAA metabolism. For instance, in Canavan's disease high levels of intracellular NAA [15] are due to aspartoacylase (ASPA) deficiency, which is the enzyme that degrades NAA to acetate and aspartate.

Further examples that show the lack of direct relationship of NAA to neuronal integrity include various pathologies such as temporal lobe epilepsy (TLE) [16] or amyotrophic lateral sclerosis (ALS) [17], which exhibit spontaneous or treatment reversals of NAA to normal levels.

Choline-containing compounds comprise signals from free choline (Cho), phosphocholine (PC) and glycerophosphocholine (GPC), with a resonant peak located at $3.22 \mathrm{ppm}$. Since the resonance contains contributions from several methyl proton choline-containing compounds, it is often referred as "total Choline" (tCho). tCho is involved in pathways of phospholipid synthesis and degradation thus reflecting a metabolic index of membrane density and integrity as well as membrane turnover [1, 2,9].

Consistent changes of tCho signal have been observed in a large number of cerebral diseases. Processes that lead to elevation of tCho include accelerated membrane synthesis of rapidly dividing cancer cells in brain tumors $[1,2,9]$, cerebral infractions, infectious diseases [18], and inflammatory-demyelinating diseases [19].

Unlike to NAA, which is distributed almost homogeneously throughout the healthy brain, tCho exhibits a marked regional variability with higher concentrations observed in the pons and lower levels in the vermis and dentate [20]. Therefore, detailed knowledge about regional variations of tCho is necessary for an accurate interpretation of the metabolite's levels, especially in diseases such as epilepsy and psychiatric disorders where tCho is subtly different to normal levels.

Creatine $(\mathrm{Cr})$ and Phosphocreatine $(\mathrm{PCr})$ together they are often referred as total creatine $(\mathrm{tCr})$ because they cannot be distinguished with standard clinical MR unit (up to 7T) and their sum is thus mentioned. $\mathrm{Cr}$ and $\mathrm{PCr}$ arise from the methyl and methylene protons of $\mathrm{Cr}$ and phosphorylated $\mathrm{Cr}$. Within the ${ }^{1} \mathrm{H}-\mathrm{MR}$ spectrum, $\mathrm{tCr}$ is located at $3.03 \mathrm{ppm}$ and $3.93 \mathrm{ppm}$ resonant frequencies.

In the brain $\mathrm{tCr}$ is present in both neuronal and glial cells and is involved in energy metabolism serving as an energy buffer via the creatine kinase reaction retaining constant 
ATP levels and as an energy shuttle, diffusing from the energy producing (i.e. mitochondria) to energy utilizing sites (i.e. nerve terminals in brain) [21]. As $\mathrm{tCr}$ is not naturally produced in the brain, its concentration is assumed to be stable with no changes reported with age or a variety of diseases and is used for calculating metabolite ratios (NAA/Cr, tCho/Cr etc) [21]. Nevertheless, the use of $\mathrm{tCr}$ as an internal concentration reference should be used with caution as decreased $\mathrm{tCr}$ levels have been observed in the chronic phases of many pathologies including tumors [22], stroke [23] and gliosis [24].

myo-inositol (mI) is a cyclic sugar alcohol that gives rise to four groups of resonances with the larger and most important signal occurring at $3.56 \mathrm{ppm}$. It is observable on short time echo (TE) spectra as it exhibits short T2 relaxation times and is susceptible to dephasing effects due to J-coupling. The exact function of $\mathrm{mI}$ is uncertain, however it has been proposed as a glial marker and an increase of $\mathrm{mI}$ levels is believed to represent glial proliferation or an increase in glial cell size, both of which may occur in inflammation [2]. Additionally, this metabolite is involved in the activation of protein $C$ kinase which leads to production of proteolytic enzymes found in malignant and aggressive cerebral tumors, serving as a possible index for glioma grading [25]. $\mathrm{mI}$ has also been labeled as a breakdown product of myelin. Thus, altered levels of $\mathrm{mI}$ have been also encountered in patients with degenerative and demyelinating diseases $[12,15]$.

Lactate and Lipids, in the normal brain should be maintained below or at the limit of detectability within the ${ }^{1} \mathrm{H}-\mathrm{MR}$ spectrum, overlapping with macromolecule (MM) resonances at 1.33ppm (doublet) and 0.9-1.3 ppm respectively. Any detectable increase in lactate and lipids can therefore be considered abnormal. Lactate is present in both intracellular and extracellular spaces and provides an index of metabolic rate and clearance [22]. As an end-product of anaerobic glycolysis, increased lactate levels have been observed in a wide variety of conditions in which oxygen supply is restricted such as in both acute and chronic ischemia [14], metabolic disorders [2], and tumors [1, 2, 9, 22]. Lactate also accumulates in tissues that have poor washout like cysts [26] and normal pressure hydrocephalus [27]. However, in CSF, lactate may be detectable at low levels in normal subjects with prominent ventricles [4].

The spectral region between $0.9 \mathrm{ppm}$ and $1.3 \mathrm{ppm}$ as referred above; represents the methylene $(1.3 \mathrm{ppm})$ and the methyl $(0.9 \mathrm{ppm})$ groups of fatty acids. It is during membrane breakdown when fractured proteins and lipid layers become visible. Regardless of the exact molecular source, an elevation of lipid resonances indicates cerebral tissue destruction such as infarction [14], acute inflammation [28] and necrosis [18]. In addition, lipid signals have been observed in patients with several metabolic disorders such as Zellweger syndrome and Refsum's disease [29].

Glutamate (Glu) and Glutamine (Gln) together they form a complex of peaks (Glx complex) between $2.15 \mathrm{ppm}$ and $2.45 \mathrm{ppm}$, as their similar chemical structures, renders their distinction difficult within a proton spectra at 1.5T. However, at 3T and above Glu and Gln start to become resolved [30] and at magnetic fields of 7T and higher, the Glu and Gln resonances are visually separated leading to big quantification accuracy [21]. Glu is the 
major excitatory neurotransmitter in mammalian brain and the direct precursor for the major inhibitory neurotransmitter, $\gamma$-aminobutyric acid (GABA). The amino acid Gln, is an important component of intermediary metabolism, is primarily located in astroglia and it is synthesized from Glu [21].

The Glx complex plays a role in detoxification and regulation of neurotransmitters. Increased levels of Glx complex are markers of epileptogenic processes [31] and low levels of Glx have been observed in Alzheimer Dementia and patients with chronic Schizophrenia [32]. Glx complex increment, has been also observed in the peritumoral brain edema correlated with neuronal loss and demyelination [33]. As reported by Malhorta et al., Glx might be used as an in vivo index of inflammation since they observed elevated Glx levels in acute MS plaques but not in chronic ones [34].

Alanine (Ala) is an amino acid present in the normal brain, resonating at $1.47 \mathrm{ppm}$. It is frequently considered as a specific metabolic charecteristic of meningiomas, however, its identification rate varies from $32 \%$ to $100 \%$ [3, 22]. It can be also presented in neurocytomas [35], gliomas and PNETs [36]. In vivo ${ }^{1} \mathrm{H}-\mathrm{MRS}$ at $1.5 \mathrm{~T}$ often cannot provide a distinction between Ala and Lac peaks as they resonate in neighboring frequencies. When both metabolites are present they produce a triplet peak located between $1.3 \mathrm{ppm}$ and $1.5 \mathrm{ppm}$ [37] observed at 3T and higher.

Glycine (Gly) is the simplest amino acid and possible antioxidant, distributing mainly in astrocytes and glycinergic neurons, where it is regulated due to its neuroactive properties as an inhibitory neurotrasmitter [28]. It resonates at $3.55 \mathrm{ppm}$ and it overlaps with $\mathrm{mI}$ rendering the observation of Gly impossible in a non-processed spectrum. In cases of $\mathrm{mI}$ absence, the even low Gly levels can be quantified [38].

High levels of Gly have been observed in glioblastomas, medulloblastomas, ependymomas and neurocytomas [28]. It has also been reported that this metabolite may provide a noticeable metabolic index for the differentiation of glioblastomas from lower grade astrocytomas, primary gliomas from recurrence [38] and glial tumors from metastatic brain tumors [36].

Taurine (Tau) gives two triplets at $3.25 \mathrm{ppm}$ and $3.42 \mathrm{ppm}$, which can be observed at higher magnetic fields [21] as they significantly overlap with Cho and mI. Tau is an inhibitory neurotransmitter that activates GABA-a receptors or strychnine-sensitive glycine receptors and it has also been proposed as an osmoregulator and a modulator of neurotransmitter action [21]. High levels of Tau have been observed in medulloblastoma, pituitary adenoma and metastatic renal cell carcinoma [39]. Shirayama et al have been also reported increased levels of Tau in the medial prefrontal cortex in schizophrenic patients [40].

Glutathione (GSH) is the major protective molecule of living cells assigned to $2.9 \mathrm{ppm}$. It serves as an antioxidant and detoxifier thus having an important role against oxidative stress [41]. Glutathione also plays a role in apoptosis and amino acid transport [42].

Altered levels of this metabolite have been reported in acute ischemic stroke patients as ischemia is associated with significant oxidative stress [41], in Parkinson's disease and other 
neurodegenerative diseases affecting the basal ganglia [21]. GSH has been also found to be significantly elevated in meningiomas when compared to other tumors [42], showing as well an inverse relationship with glioma malignancy.

Several other amino Acids such as Succinate at $2.4 \mathrm{ppm}$, Acetate at $1.92 \mathrm{ppm}$, Valine and Leucine at $0.9 \mathrm{ppm}$ together with Alanine and Lactate, are the major spectral findings of bacterial and parasitic diseases. Acetate and Succinate are presumably originating from enhanced glycolysis of the bacterial organism [9]. The amino acids Valine and Leukine are known to be the end-products of proteolysis by enzymes released in pus [9]. Specifically, Leucine and Valine peaks have been detected in cystercercosis lesions, however they have not been reported in proton MR spectra of brain tumors [9].

\section{Technical considerations}

In order to precisely identify the metabolite peaks within a spectrum, several technical considerations should be taken into account concerning the applied magnetic field, the shimming procedures as well as the adequate voxel positioning and the available ${ }^{1} \mathrm{H}-\mathrm{MRS}$ techniques, which all highly affect the quality of the yielded spectrum before any postprocessing intervention.

\subsection{Field strength}

In ${ }^{1} \mathrm{H}-\mathrm{MRS}$ clinical applications, it is not the signals of water and fat that are of interest, but rather the smaller signals of metabolites, thus a magnetic field of sufficient strength is required. Therefore, most clinical ${ }^{1} \mathrm{H}$-MRS measurements are performed using MR systems with field strengths of $1.5 \mathrm{~T}$ and higher. Although more powerful 4-, 6-, 7- , and even 8T MR body scanners are currently in use, the most common high field systems operate at 3T. The main advantage of increasing magnetic field strength is the subsequent increase of the signal-to-noise ratio (SNR). Theoretically, SNR increases proportionally to field strength, however, when put into clinical practice, the study of Barker et al [43], demonstrated a $28 \%$ increase in SNR at 3T compared to that of 1.5T at short TEs, appreciably less than the theoretical $100 \%$ improvement. Another advantage of magnetic field increment, is the proportional increase of the Chemical Shift, from $220 \mathrm{~Hz}$ at $1.5 \mathrm{~T}$ to $440 \mathrm{~Hz}$ at 3T. This is reflected by more effective water suppression and improved baseline separation of $\mathrm{J}$ coupled metabolites such as glutamate, glutamine and GABA, without the need of sophisticated spectral editing techniques [44]. The improvement in spectral resolution is further evident at 7T where weakly represented neurochemicals with important clinical impact, such as scyllo-Ins, aspartate, taurine and NAAG, can be clearly visible [44].

On the other hand, the aforementioned advantages may be hampered by intrinsic fielddependent technical difficulties that should be considered. When the frequency shift between two adjacent nuclei is large enough, a measurable alteration of MR signal, used to encode the $\mathrm{x}$ - and $\mathrm{y}$-axis spatial coordinates, will occur producing a spatial misregistration. This means that the volume of MRS information may not be the same as that displayed on the localizer MR image [45]. J-modulation anomalies represent another difficulty 
encountered at high magnetic fields. The large separation of coupled resonances such as Lactate can result in incomplete inversion of the coupled spin over a large portion of the selected volume, resulting in anomalous intensity losses at long echo times. Strategies to quantify the lactate signal loss have been previously discussed by Lange et al. [46]. Magnetic susceptibility from paramagnetic substances and blood products, are sensibly increased with increasing magnetic field strength. Consequently, magnetic field inhomogeneity and susceptibility artifacts makes more difficult to obtain good-quality spectra, especially from largely heterogeneous lesions [45]. Improved local shimming methods can alleviate the problem.

\subsection{Shimming}

Shimming refers to the process of adjusting field gradients, either manually or automatically, in order to optimize the magnetic field homogeneity over the volume under study. Magnetic field inhomogeneities result primarily from susceptibility differences between different tissues and between tissue and air cavities, which are scaled non-linearly in ultra-high magnetic fields [47]. Thus, voxels that are placed in inhomogeneous regions of the brain, such as the temporal poles, are difficult to shim due to their close proximity to the sinuses.

Field homogeneity is specified by measuring the full width at half maximum (FWHM) of the water resonance, which determines the spectral resolution. Special emphasis, especially when field is increased, must be placed on shimming, as it increases both sensitivity and spectral resolution. This is why most devices come equipped with second or third order shimming by monitoring either the time domain or frequency domain of the ${ }^{1} \mathrm{H}-\mathrm{MRS}$ signal [48]. Some times 4-order shimming might be necessary [49], especially in cases when field homogeneity should be reached in large volumes of interest during magnetic resonance spectroscopic imaging (MRSI).

Effective shimming requires methods for mapping field's strength variations over the area under study. Methods that have been developed for field mapping can be grouped in two categories: those which are based on 3D field mapping [49] and those which map the magnetic field along projections [50]. In both shimming methods, information about the magnetic field variation is calculated from phase differences acquired during the evolution of the magnetization in a non-homogeneous field.

\subsection{Voxel positioning}

For a meaningful in vivo ${ }^{1} \mathrm{H}-\mathrm{MRS}$, it is important to locate the voxel in the appropriate region for a reliable metabolic characterization of a lesion [48].

First and foremost, cautious spatial localization is used to remove unwanted signals from outside the ROI, like extracranial lipids and to avoid "partial volume effects", thereby providing a more genuine tissue characterization. Additional benefits from careful spatial voxel localization, originate from the fact that variations in the main magnetic field and 
magnetic field gradients, are greatly reduced, thereby providing narrower spectral lines and more uniform proton excitation.

Several lesions and stroke infarcts do not always place themselves in positions that are easy to shim such as temporal lobes, the base of the brain and the cortex near the scull. Small voxels is those regions are easier to shim, but the signal also depends on volume so a voxel with $1-\mathrm{cm}$ sides is often considered the practical minimum size to achieve a reasonable SNR [51].

\section{4. ${ }^{1} \mathrm{H}-\mathrm{MR}$ spectroscopy data acquisition techniques}

Spectra can be acquired either with a single voxel (SV) technique (single voxel spectroscopy, SVS) or multiple voxels technique, known as either magnetic resonance spectroscopic imaging (MRSI) or chemical shift imaging (CSI) in two or three dimensions. SVS is based on the stimulated echo acquisition mode (STEAM) [52] or the point resolved spectroscopy (PRESS) [53] pulse sequences while MRSI uses a variety of pulse sequences (Spin Echo, PRESS etc.) [54].

SVS acquires a spectrum from a small volume of tissue located at the intersection of three mutual orthogonal slice-selective pulses as depicted in figure 2. The pulse sequence is designed to collect only the echo signal from the point where all three slices intersect [53].
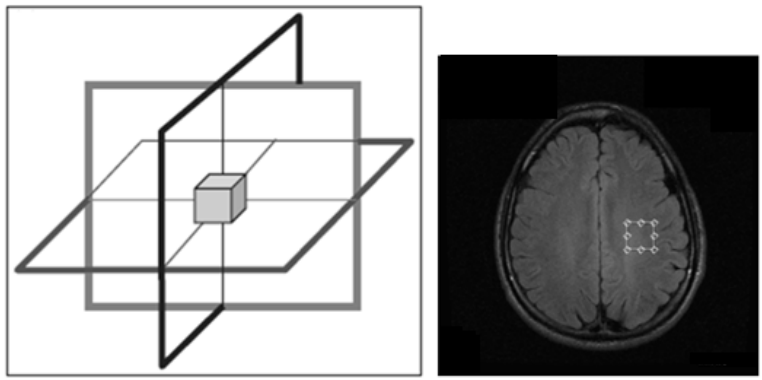

Figure 2. Schematic representation of the three orthogonal SV slice selective pulses (left) resulting in the signal collection only from the rectangular region of interest.

The advantages of this approach are that:

1. the volume is typically well-defined with minimal contamination (e.g. extracranial lipids),

2. the magnetic field homogeneity across the volume can be readily optimized, leading to

3. improved water suppression and spectral resolution.

The main disadvantage of SVS is that it does not address spatial heterogeneity of spectral patterns and in the context of brain tumors, these factors are particularly important for treatment planning such as radiation or surgical resection.

Lesion's heterogeneity is better assessed by MRSI. MRSI techniques have been extended to two dimensions (2D) by using phase-encoding gradients in two directions, or, subsequently, 
three-dimensional (3D) encoding [55]. Thus, the detection of localized ${ }^{1} \mathrm{H}-\mathrm{MR}$ spectra from a multidimensional array of locations is allowed (Figure 3). While technically more challenging -due to (1) significant magnetic field inhomogeneity across the entire area of interest, (2) spectral degradation due to intervoxel contamination the so called "voxel bleed", (3) long data acquisition times and (4) post-processing of large multidimensional datasets- MRSI can detect metabolic profiles from multiple spatial positions, thereby offering an unbiased characterization of the entire object under investigation.
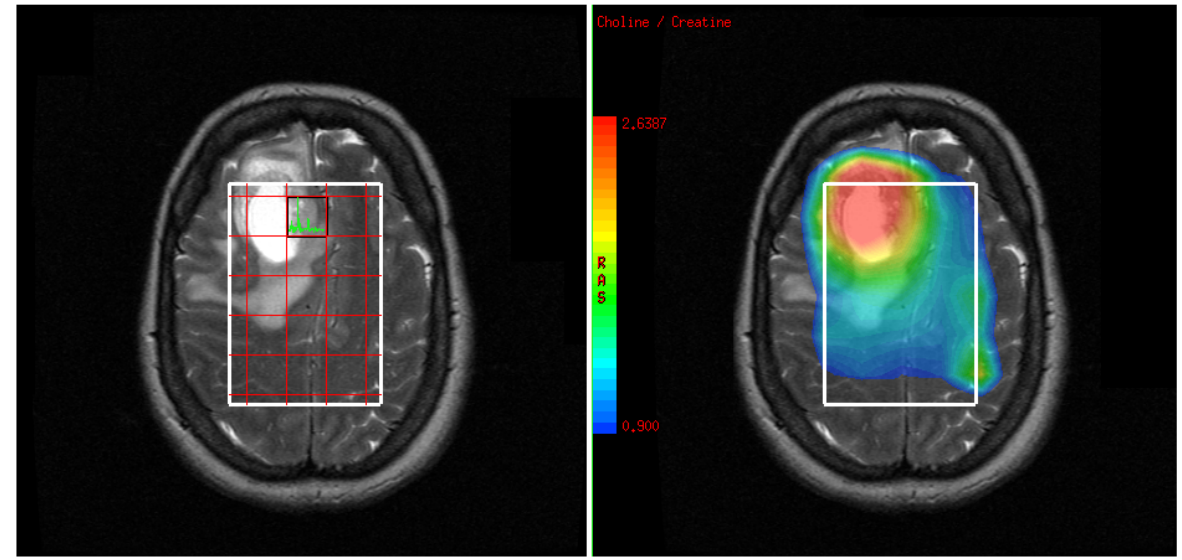

Figure 3. An example of 2D-MRSI of a 50-year old female with a glioblastoma. Simultaneously acquired spectra from multiple regions located at the same plane of the lesion (left). Data are also presented as a metabolic map of Choline/Creatine (right).

\subsection{Water and lipid suppression techniques}

Water and peri-cranial lipid suppression techniques are of paramount importance in ${ }^{1} \mathrm{H}$ MRS procedure in order to observe the much less concentrated metabolite signals. The metabolites of interest are usually about a factor of 8,000 less in concentration than water. Therefore, the water suppression efficiency should be robust and should not vary spatially across the field of view (FOV).

The existing water suppression techniques can be divided into three major groups, namely: (1) methods that employ frequency-selective excitation and/or refocusing pulses; or (2) utilize differences in relaxation parameters; and (3) other methods, including software-based water suppression. The most common method of the first group utilizes multiple (typically 3) frequency-selective, $90^{\circ}$ pulses (chemical shift selective water suppression (CHESS) pulses [56], prior to localization pulse sequence. Additionally suppression can be achieved by selectively diphase water, while metabolites of interest are rephased using refocusing pulses during the spin echo period [57]. As water and metabolites T1s are sufficiently different, it is possible to suppress the water signal and observe the metabolites in the close proximity to the water resonance [58]. The third method involves the acquisition of two separated scans in which the metabolite resonances are inverted. The large (unsuppressed) water resonance, 
as well as the water-related sidebands, is not inverted in either scan. The difference between the two scans therefore results in a water-subtracted (suppressed) metabolite spectrum without any interfering water-related sidebands [21].

Lipid suppression can be performed by avoid the excitement of the lipid signal using STEAM or PRESS localization to select a relatively large rectangular volume inside the brain. Since the extracranial lipids are not excited they do not contribute to the detected signal. Opposite to the strategy employed by volume pre-localization, outer volume suppression pulses (OVS) are applied to presaturate the lipid signal [54]. As illustrated in figure 4, rather than avoiding the spatial selection of lipids, OVS excites narrow slices centered the brain's lipid-rich regions. Additionally, the difference in T1s of lipids (250-350 $\mathrm{msec})$ and metabolites $(1000-2000 \mathrm{msec})$ allows the application of an inversion pulse (inversion time $\sim 200 \mathrm{msec}$ ), which will selectively null the lipid signal [59]. By choosing the inversion delay such that the longitudinal lipid magnetization is zero, the lipids are effectively not excited.
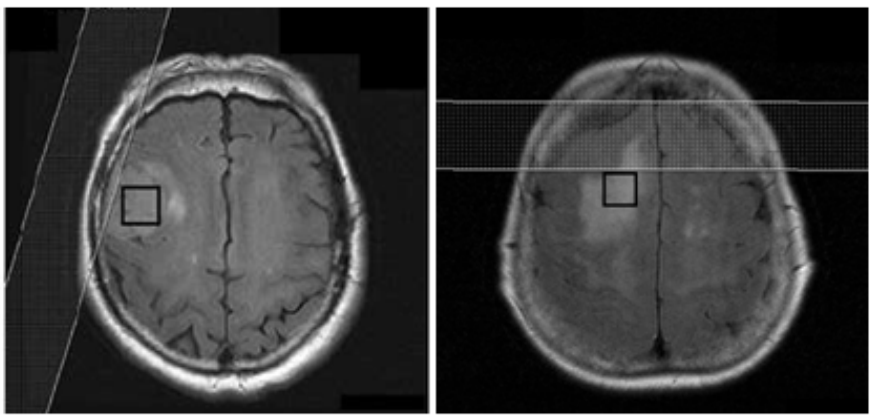

Figure 4. The location and orientation of OVS pulses have been prescribed in order to saturate as much peri-cranial lipid as possible while the signal within the voxel remains unperturbed.

\section{Post processing techniques}

In MR spectroscopy, post-processing is considered any signal manipulation performed in order to improve the visual appearance of the MR spectrum or the accuracy during metabolite estimation. Therefore, for a reliable analysis of in vivo ${ }^{1} \mathrm{H}-\mathrm{MR}$ spectra, an understanding of the principles of post-processing techniques is necessary.

Signal post-processing can be performed either on time domain or after Fourier transformation on frequency domain [60]. Eddy current correction, removal of unwanted spectral components, signal filtering, zero filling, phase correction and baseline correction, consist the most common post-processing techniques for effective signal improvement, and they will be briefly discussed below:

During signal localization RF pulses are applied together with magnetic field gradients. The switching pattern of the gradients applied, can cause eddy current (EC) artifacts that are time and space dependent, causing time dependent phase shifts in the FID and distorted 
metabolite lineshapes within the spectrum preventing accurate quantification. In a spectrum EC artifacts can be removed by acquiring an additional FID without water suppression. The phase of the water FID is determined in each time point and it is subtracted from the phase of the corrupted FID [24]. The EC artifact correction comprises the first step of the postprocessing procedure.

The removal of unwanted signals from the FID which may disturb signals from the resonances of interest is the next step of signal post processing. A typical example of such an unwanted signal in ${ }^{1} \mathrm{H}-\mathrm{MRS}$ is that of water. Water suppression during measurement is never perfect and a residual water signal remains in the spectrum which often has a complicated lineshape [24]. Residual water elimination from the FID can be achieved, either by approximating the water signal and subtract it from the FID, or by eliminate it using special filters [61], or by applying baseline correction for the removal of the broad water peak from the spectrum [62].

The existence of a distorted spectral baseline hampers quantitative analysis as the estimation of metabolite peak areas is not reliable. The main sources of the baseline signal are fast decaying components with very short $\mathrm{T}_{2}^{*}$ values such as macromolecules, hardware imperfections, signal from the sample and as mentioned above, inefficient water suppression. Thus, for robust data acquisition and quantification methods, baseline correction is of paramount importance. Delayed acquisition (e.g. TE $>80 \mathrm{~ms}$ ) removes the macromolecules due to their shorter T2 relaxation times ( $30 \mathrm{msec})$, at the expense of loss of information of many scalar-coupled resonances [21] which have been suggested valuable for tumor and stroke characterization [4, 21, 22, 24, 25, 33].

Special functions, called filters, can be subsequently applied at the signal in the time domain. The goal is to enhance or suppress different parts of the FID leading to improved signal quality. The three most commonly used filtering approaches are: sensitivity enhancement, to reduce the noise from the FID; resolution enhancement, to achieve narrower metabolite linewidths; and apodization for signal's ripple (due to signal truncation) reduction [62].

The FID of a spectrum, when acquired, is sampled by the analog-to-digital converter over $\mathrm{N}$ points in accordance to the Nyquist sampling frequency. Therefore, if the number of points is not sufficient, the reliable representation of the signal fails. Instead of increasing the acquisition time with the inevitable noise increment, the acquired FID can artificially be extended by adding a string of points with zero amplitude to the FID prior to Fourier Transformation, a process known as zero filling. Zero filling does not increase the information content of the data but it can greatly improve the digital resolution of the spectrum and helps to improve the spectral appearance [21], rendering it an important postprocessing step.

After Fourier transformation, the spectrum will be phase corrected. When the zero-phased FID signal shifts to the frequency domain, yields a complex spectrum with absorption (real) and dispersion (imaginary) Lorentz peaks. However, when the initial phase is non-zero, it is not attainable to restore pure absorption or dispersion line shapes and phase correction 
must be applied [4, 21, 62]. A zero-order phase correction compensates for any mismatch between the quadrature receive channels and the excitation channels to produce the pure absorption spectrum, whereas, a first-order phase correction compensates for the nuclei dephase due to the delay between excitation and the detection of FID [62].

\section{6. ${ }^{1} \mathrm{H}-\mathrm{MRS}$ metabolic profiles of brain lesions}

The effective differential diagnosis of brain lesions using ${ }^{1} \mathrm{H}-\mathrm{MRS}$ depends on the ability of the experienced neuroscientist to interpret and evaluate the metabolic criteria and data underlying each disease. However, similarities in the chemical composition among diseases and/or atypical metabolic characteristics, often burden the diagnosis. Thus, a clinical guide to the main MR spectroscopic findings of cerebral disorders is necessary.

This section focuses on the metabolic patterns of a variety of intra-cranial diseases.

\subsection{Multiple Sclerosis (MS)}

Multiple Sclerosis (MS) is an auto-immune inflammatory disease of the central nervous system (CNS) in which the myelin sheaths around the axons are damaged leading to demyelination, neuronal affection, inflammation, gliosis and axonal degeneration [14]. ${ }^{1} \mathrm{H}$ MRS is particularly informative in MS, by providing evidence of the two primary pathologic processes of the disease: active inflammatory demyelination and neuronal injury in both lesional and non-lesional brain tissue [63, 64].

Acute demyelinating lesions reveal increased Cho and Lac resonance intensities due to the release of membrane phospholipids during active myelin breakdown and the impaired metabolism of the inflammatory cells, respectively [63]. Short TE spectra also provide evidence of increased lipids, $\mathrm{mI}[63,64]$ and glutamate levels [34]. Increased glutamate levels in acute MS lesions address a link between the direct axonal injury and glutamate excitotoxicity [65], whereas $\mathrm{mI}$ is suggestive of glial proliferation and astrogliosis [63]. The aforementioned changes are accompanied by a substantial decrease in NAA due to axonal injury reflecting metabolic or structural changes [64, 65]. It is important to note that the spectroscopic changes seen in acute MS plaques are often very similar to the spectra observed in brain tumors (high Cho, low NAA, increased Lac, etc.), and therefore this should be kept in mind when evaluating spectra from patients with undiagnosed brain lesions.

After the acute phase transition, Lac, Cho and lipids seem to return to normal levels, whereas NAA may remain decreased or show partial recovery, lasting for several months [64]. The recovery of NAA can be attributed to resolution of edema, diameter increment of the previously shrinked axons, as a result of the re-myelination and reversible metabolic changes in neurons [64, 65]. There are reports of elevated Cho resonance in chronic MS plaque, probably reflecting the associated gliotic process [66]. Cr seems to be a variable metabolite both in chronic and acute, but is also described to be slowly increasing over time, indicative of gliotic reaction or attempts of incomplete re-myelination of the chronic diseased tissue phases [14]. 
Metabolic abnormalities in MS patients not only concern the lesions, but are found throughout the normal appearing white matter (NAWM) with notably reduced NAA, which is thought to indicate diffuse axonal dysfunction or loss. It must also be stressed out that the presence of intense gliosis may also cause increased levels of $\mathrm{mI}$ [67] and Cr [68]. Increased glutamate, lipids and Cho can be also found in regions of the NAWM, which later are going to develop T2-hyperintense focal lesions [64].

\subsection{Intracranial abscesses}

Brain abscesses are focal, intracerebral infections that begin with a localized region of cerebritis, evolving into a discrete collection of pus surrounded by a well-vascularized capsule. The causative organisms involved in brain abscesses are quite variable, and may consist of mixed cultures: aerobes, anaerobes, facultative anaerobes, and facultative anaerobes in combination with aerobes/anaerobes.

MRS has been proven beneficial in differentiating between brain abscesses and other cystic lesions [69], which can be used to implement the appropriate antimicrobial therapy. Brain abscesses reveal specific metabolic substances, such as succinate, acetate, alanine, valine, pyrouvate, leukine, lipids and lactate $[69,70]$,which are all present in untreated bacterial abscesses or soon after the initiation of treatment [70]. Increases in lactate, acetate, and succinate presumably may originate from the enhanced glycolysis and fermentation of the infecting microorganisms. Amino acids such as valine and leucine are known to be the end products of proteolysis by enzymes released by neutrophils in pus [14]. However, cerebral abscesses contain no neurons [71], therefore no peaks of NAA and $\mathrm{Cr} / \mathrm{PCr}$ should be detected. The detection of any NAA and/or $\mathrm{Cr} / \mathrm{PCr}$ is indicative of either signal contamination or erroneous interpretation of acetate peak as NAA [72]. Similarly no tCho peak is present in an abscesses spectrum because there are no membranous structures in its necrotic core [73]. On the other hand, abscesses of tuberculous origin are characterized by the predominant presence of lipids, moderate increase of tCho resonance and no evidence of cytosolic amino acids [4].

Differential diagnosis of brain abscess versus brain tumor is sometimes difficult on the basis of imaging findings and clinical judgment, especially in the case of a brain tumor with a mainly cystic or necrotic component. However, because the vast majority of the aforementioned amino acids have not been detected in brain neoplasms, their presence strongly differentiates abscesses from highly aggressive tumors [71].

\subsection{Ischemia}

Most studies of ${ }^{1} \mathrm{H}-\mathrm{MRS}$ of the human brain have focused on the signals from NAA and lactate, as potential markers of brain ischemia, respectively, although there are also often changes in the other metabolite signals, such as Cho, Cr, glutamate (Glu) and glutathione (GSH) [74]. The time course of these metabolite changes through time is an important factor for the diagnosis and prognosis of a brain infarct. 
In acute stroke the infarct core rapidly shows signs of cell death and a spectrum from this area has the characteristic lactate peak, often with a broad lipid peak too. Lactate could also arise from a shift toward anaerobic glycolysis in potentially viable cells that continue to metabolize glucose under locally hypoxic conditions [75]. Lactate may also be present in smaller concentrations in the ischemic penumbra, the region around the core which if quickly re-perfused may recover its function [75]. Lactate formed in the initial period of ischemia could remain in necrotic tissue and leave the region of injury after cell lysis in a period of weeks or months after the stroke onset.

Unlike to the increase of lactate, NAA is observed to slowly decrease over a time scale of hours after the induction of ischemia [75]. Several studies have described an initial rapid decrease in NAA of about $10 \%$ within the first few minutes followed by a slower decrease. It has been suggested that NAA diminish may be due to NAA degradation by enzymes within the injured neurons in the first few days or hours following infarction, or perhaps due to changes in other molecules (e.g. Glu, Gln, GABA etc.) which overlap with the spectral resonance of NAA [74].

tCho has been observed to either increase or decrease both in acute and chronic human ischemia [76]. Increases in Cho in stroke may be the result of gliosis or ischemic damage to myelin, while decreases are probably the result of edema, necrosis and cell loss [4]. Initial reduction in $\mathrm{Cr} / \mathrm{PCr}$ is identified following infarction and further reductions have been demonstrated up to ten days following the time of onset [74]. Muniz Maniega et al. reported continuous reduction of $\mathrm{Cr}$ levels over a period of three months from the stroke onset [76].

A study by Rumpel et al. revealed that $\mathrm{mI}$ might also significantly contribute to the understanding of brain tissue response to ischemia, which is in line with a persistent cytotoxic swelling, attributed to the glial population, found in early subacute ischemic infarcts [77]. Acute ischemic also causes changes in the glutathione (GSH) system (decreased GSH in ischemic patients) as stroke is associated with significant oxidative stress [41]. Experimental studies have suggested that ischemic stroke may cause an increment in extracellular level of GABA; however there is very little work on the detection of GABA and glutamate in cerebral ischemia [78].

\subsection{Epilepsy}

The term epilepsy covers a wide group of syndromes with varied etiology and prognosis. By providing an insight into the biochemical processes related to epileptic seizures, ${ }^{1} \mathrm{H}$-MRS aids in the localization or lateralization of the epileptogenic foci and in the influence of the metabolites concentration after the administration of antiepileptic drugs and/or after resection of the epileptogenic tissue.

Temporal lobe epilepsy (TLE) associated with hippocampal sclerosis (HS) is the most common refractory focal epilepsy. The localization is performed by the comparison of metabolites on the left and right temporal lobe, especially in the hippocampus and temporal poles, to determine which hemisphere is responsible for the genesis of seizures [79]. The 
metabolites of interest in epilepsy are NAA, GABA and glutamine/glutamate (Glx) and the less prominent $\mathrm{mI}$ and lactate (Figure 5). Most of the studies dealing with mesial TLE, demonstrate decreased levels of NAA in the affected temporal lobe when compared with controls or with the homologous non-epileptic contralateral region, with no changes or mild increases of tCho. Interestingly, not only decrease of NAA content occurs in the epileptogenic foci, but also unilateral presence of lactate in the mesian temporal lobe could potentially be indicative of the side of the epileptogenic zone [16].

Nowadays, a hypothesis exists in which the raise of mitochondrial energy consumption promotes a reduction of neuronal synthesis of NAA, and, therefore, an increase of glutamate (its precursor) [80]. In epileptic patients, it seems to exist a disequilibrium of glutamine/glutamate (Glx) and GABA [81]. Therefore, spectroscopic measurements of Glx complex could yield spatial information on the epileptogenic zone. However, although there is some evidence that Glx is elevated in TLE, its value as a marker for the epileptogenic zone has not been established yet.

Additionally, ${ }^{1} \mathrm{H}-\mathrm{MRS}$ studies of TLE have also been focused on $\mathrm{mI}$, however, its role remains controversial. The study of Wellard et al. revealed elevated $\mathrm{mI}$ in the epileptogenic temporal lobe of patients with HS [82]. They also reported a difference of $\mathrm{mI}$ levels between the seizure focus (temporal lobe) where $\mathrm{mI}$ is increased, and areas of seizure spread (frontal lobe) where $\mathrm{mI}$ is decreased. Thus, ${ }^{1} \mathrm{H}$-MRS may aid to the distinction of primary epileptogenic brain damage from seizure secondary effects on adjacent normal brain and help to distinguish drug refractory TLE patients, who will benefit from surgery by predicting postoperative outcome [83].

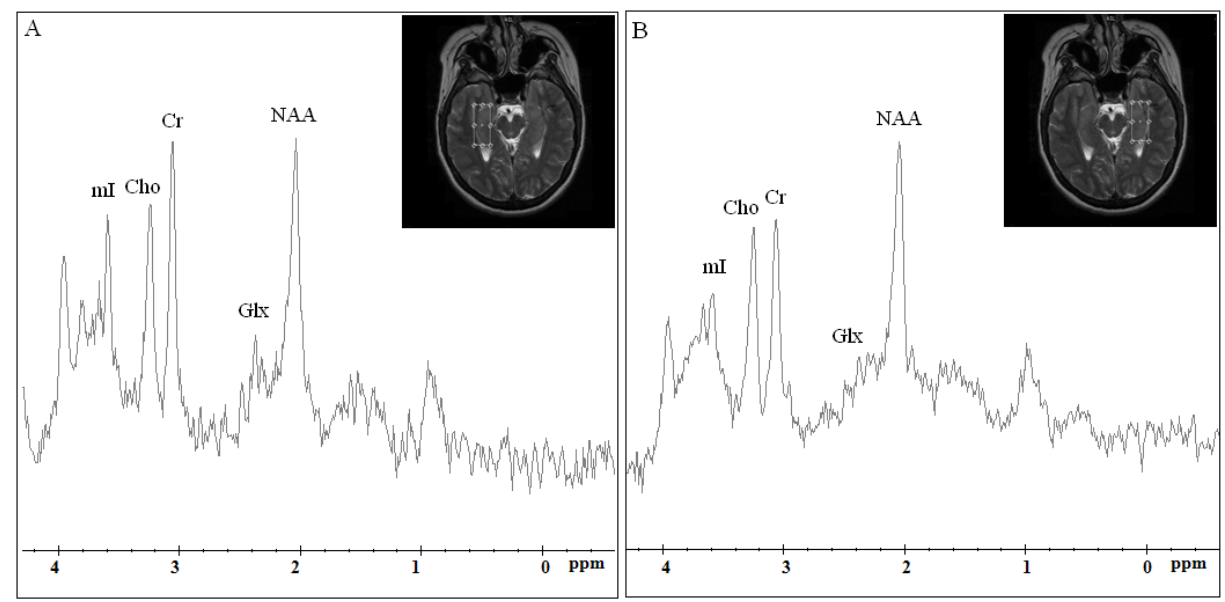

Figure 5. Short TE (35msec) spectra at $3 \mathrm{~T}$ obtained in the left and right hippocampal formation from a patient with right HS using single-voxel technique. The decreased NAA signal and the increased $\mathrm{mI}$ at the affected region (A) are evident when compared with the contralateral normal hippocampal formation (B). Note the mild elevation of the Glx complex at the affected region (B). 


\subsection{Neurodegenerative diseases (Alzheimer/Parkinson)}

Numerous studies have attempted to identify specific metabolic markers for different neurodegenerative diseases, such as Alzheimer's dementia (AD) and Parkinson's disease (PD), which concern loss of structure or function of neurons including death of the neuronal cells. The clinical objective in that cases is to establish a precise and early diagnosis as well as to understand the related brain changes that could help to slow down the course of the disease [60].

${ }^{1} \mathrm{H}$-MRS has been demonstrated to be highly specific and sensitive to the diagnosis of Alzheimer's dementia (AD) [15]. Reduction in NAA is the most frequent ${ }^{1} \mathrm{H}-\mathrm{MRS}$ finding in AD $[84,85]$. Single Voxel ${ }^{1} \mathrm{H}-\mathrm{MRS}$ studies have consistently found reductions in NAA/Cr ratio in the hippocampal formation [85] as well as other temporal regions [86] and the posterior cingulate gyrus [87]. Findings of reduced NAA have been also detected in the temporoparietal area, and the occipital lobes $[85,87]$. There have been conflicting reports regarding Cho levels in patients with $\mathrm{AD}$. Some researchers found elevated Cho and/or $\mathrm{Cho} / \mathrm{Cr}$ ratios in $\mathrm{AD}$ patients, while others not [32, 85, 87]. Increased $\mathrm{mI}$ has been also observed, most often, in the temporal-parietal area [85], the posterior cingulate gyrus [32, 86, 87], the parietal white matter 86] and less often in the frontal lobes [86]. Few studies have also reported reduced Glx levels in $\mathrm{AD}$ patients compared to control subjects in the posterior cingulated gyrus [88] and lateral temporal cortex [86].

The majority of 'H-MRS studies in Parkinson's Disease (PD) to date have primarily targeted brain levels of NAA, Cr, and Cho [89, 90]. Many researchers disclosed a significant reduction of ratios $\mathrm{NAA} / \mathrm{Cr}$ and NAA/Cho in the temporoparietal cortex [91], the substantia nigra, the basal ganglia [92], the striatum or the occipital lobe [93]. Griffith et al. have demonstrated lower NAA/Cr ratios in the posterior cingulate gyrus of demented versus non demented subjects with PD [94]. Other investigators, however, have not detected such changes [95] in NAA, Cr, and Cho measurements, and the reasons for these different findings need to be resolved.

\subsection{Gliomas}

Gliomas are spatially heterogeneous lesions which arise from the 'gluey', or supportive tissue of the brain. The main types of gliomas are astrocytomas, oligodendrogliomas, and ependymomas. ${ }^{1} \mathrm{H}$-MRS is increasingly used in clinical studies to non-invasively identify regions with metabolic specific characteristics that reflect glioma type and grade.

A common observation in ${ }^{1} \mathrm{H}-\mathrm{MRS}$ of all glial tumors is a decreased levels of NAA and increased levels of tCho with a significant overlap among different glioma types [2, 22]. Thus, ${ }^{1} \mathrm{H}-\mathrm{MRS}$ is currently used primarily to differentiate glial tumor grade rather than to confirm a histopathological diagnosis [96].

However, the signal intensity of glutamine and glutamate (Glx) may aid the distinction between oligodendrogliomas and astrocytomas. Rijpkema et al. found significantly increased Glx levels for oligodendrogliomas when compared to that of astrocytomas [97] 
using short TE ${ }^{1} \mathrm{H}-\mathrm{MRS}$. Additionally, in a study by Majos et al, ependymomas differentiated well from the other glial tumors by showing prominent peaks of mI+Gly and Taurine at long TE spectra [98].

Discrimination between tumor grades in gliomas is an important clinical issue, because there is a dispute on the optimum treatment strategy for patients with low-grade tumors. It remains an open question whether ${ }^{1} \mathrm{H}$-MRS is able to define WHO grade of gliomas. However, a recent study by Porto et al. revealed a more prominent loss of NAA and increase of tCho in WHO III over WHO II astrocytomas [99]. They consequently proposed $\mathrm{NAA} / \mathrm{tCho}$ ratio as the most accurate index to discriminate between those tumor grades which is in agreement with what it is generally accepted, i.e. NAA/tCho ratios decrease with higher histological grade of gliomas. Law et al. demonstrated a threshold value of 1.6 for tCho/NAA which provided $74.2 \%$ sensitivity and $62.5 \%$ specificity in predicting the presence of a high-grade glioma [100]. Thus it is obvious that there is a consistent correlation between Cho increase as well as NAA decrease and tumor grade.

A study by Moller-Hartmann et al. revealed that instead of tCho, the amount of lipids proved to be the second-best discriminator between low- and high-grade gliomas, with glioblastomas multiforme (GBM) to exhibit the highest amount of lipids since necrosis is one of their microsopic hallmarks [3]. Although it has been previously proved that lactate also increases with grade, it is not always significantly differentiated between high and low grade gliomas [22]. Poor correlation between tumor grade and lactate is most likely due to the difficulty of accurately quantifying lactate in the presence of high lipid signals.

Short TE studies have also shown that $\mathrm{mI}$ levels may aid tumor classification and grading $[22,25]$. Specifically, Castillo et al. retrospectively studied 34 patients with astrocytomas and found a trend towards lower $\mathrm{mI}$ levels in high-grade compared with low-grade tumors [25].

One of the most interesting results of the study by Server et al. was the elevation in the peritumoral $\mathrm{Cho} / \mathrm{Cr}$ and $\mathrm{Cho} / \mathrm{NAA}$ metabolite ratios in relation to glioma grading [101]. Thereby, as gliomas are infiltrating intracerebral tumors, ${ }^{1} \mathrm{H}-\mathrm{MRS}$ may allow to readily appreciate their grade in the perifocal region.

\subsection{Cerebral metastasis}

Cerebral metastases are a common complication of cancer and can affect $20 \%$ to $40 \%$ of patients [102] who suffer from primary tumors in lung, breast, skin or colon.

When a metastatic brain tumor presents as a solitary lesion, it is usually indistinguishable from a high grade glioma [103]. Their distinction is important because the treatment approach and follow-up are different for these two different tumors.

The potential of in vivo ${ }^{1} \mathrm{H}$-MRS for differentiating intracerebral metastases from GBMs has been investigated in a number of studies [102, 104]. Older studies [22, 105] have reported that intratumoral ${ }^{1} \mathrm{H}-\mathrm{MRS}$, either on short or long TE, was unable to differentiate between metastases and GBMs, as they share common metabolic features. Those concern increased levels of lipids and tCho and reduced levels of NAA as it is depicted in figure 6 . 
Nevertheless, a study by Moller-Hartman et al. revealed elevated lipids for metastases, with statistically significant difference from GBMs [3]. Opstad et al. speculated that the differences in lipid profiles may be related to differences of membrane structures of infiltrative versus migratory tumor cells [106]. Significantly higher $\mathrm{Cho/Cr}$ ratio for metastases than for GBMs was reported by Server et al. due to GBMs higher levels of necrosis [102]. On the contrary, Law et al. revealed significantly lower Cho/Cr ratio for metastases than for high grade gliomas [105]. These conflicting results may be due to the intrinsic heterogeneity of such tumors.
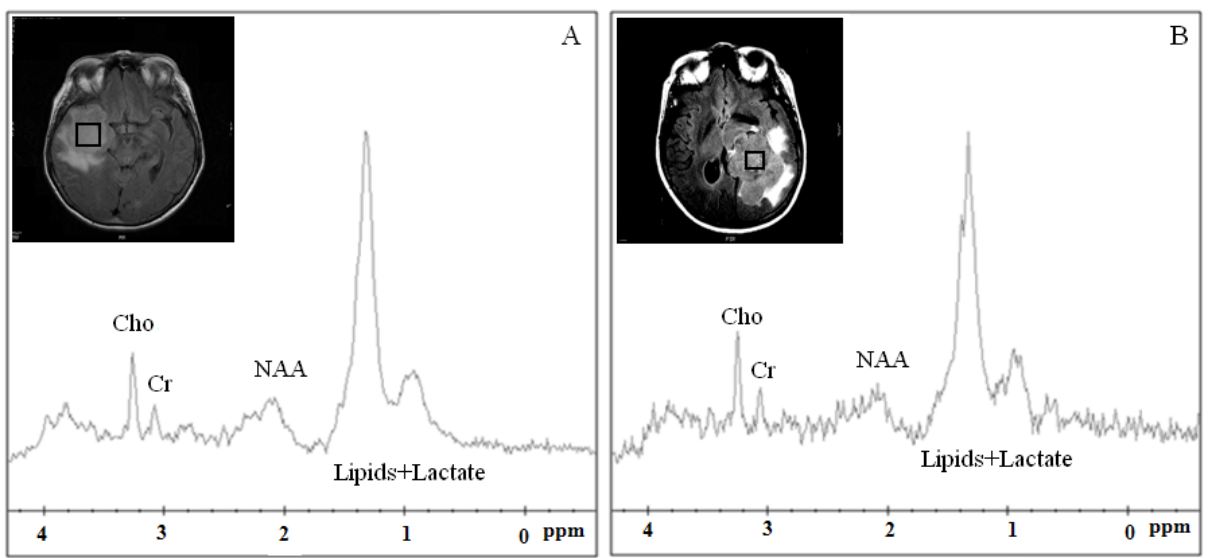

Figure 6. Typical Short TE spectra from glioblastoma multiforme (A) and intracerebral metastases (B).

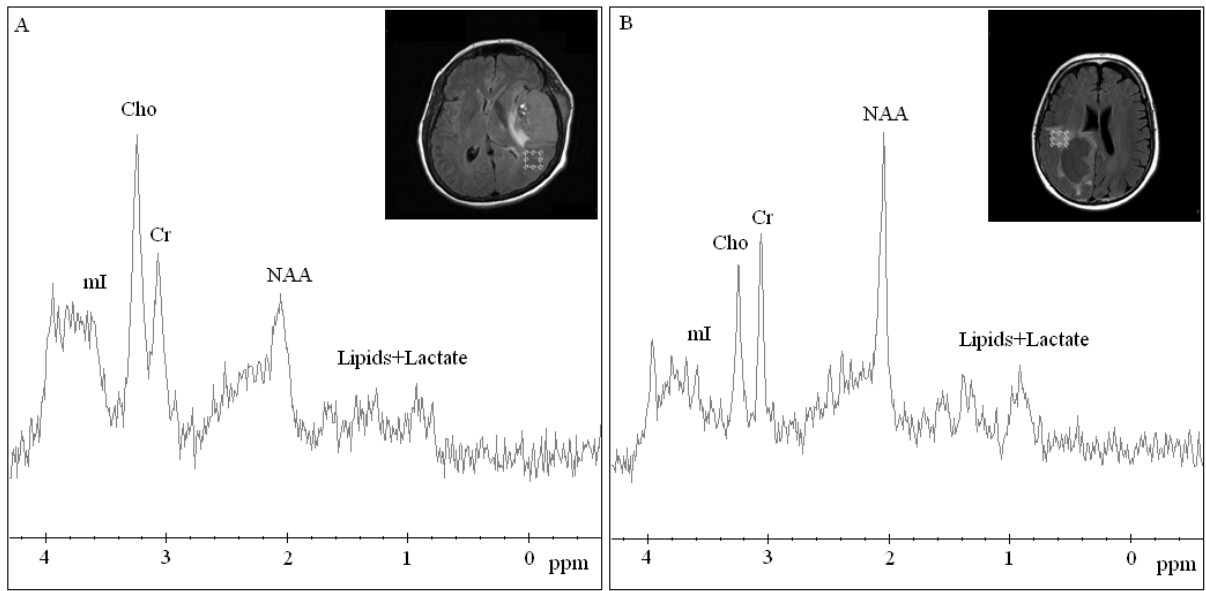

Figure 7. Short TE spectra from peritumoral areas of glioblastoma multiforme (A) and intracerebral metastases (B). 
Furthermore, promising results in differentiating between GBMs and metastases by means of the resonances at $3.56 \mathrm{ppm}$, represented by the sum of $\mathrm{mI}$ and Gly, have been previously observed [36]. Gly/mI showed a tendency to be higher in GBMs than in metastases.

Measuring the peritumoral metabolites or metabolic ratios is often more useful in differentiating intracranial metastases from high grade gliomas with more reproducible results among different studies. Elevated Cho as well as reduced NAA have been found in the peritumoral region of high-grade gliomas, but not in the peritumoral region of metastases when compared to normal levels as it is illustrated in figure 7.

Those findings support the hypothesis that the edema surrounding metastases is purely vasogenic, while the peritumoral region of GBMs is characterized by extensive infiltration of tumor cells [102, 104-106].

Some patients get their brain metastases detected before the primary cancer. Since GBM case has being withdrawn from the differential diagnosis, identification of metastases type would be important for further treatment. Sjobakk et al. investigated the feasibility of using ${ }^{1} \mathrm{H}-\mathrm{MRS}$ to characterize brain metastases originating from different primary cancers. The results presented in their study, demonstrated that lipid signals on both short and long TE spectra are important for metastases characterization. Although non-statistically significant, lung metastases tended to differentiated from breast metastases in respect to their lipid signals, while the melanoma showed no trend [107]. Chernov et al retrospectively studied 25 metastatic brain tumors from lungs, colon, breast, kidney, prostate and cardiac muscle, using ${ }^{1} \mathrm{H}-\mathrm{MRS}$ on long TE. The detected metabolic characteristics revealed that metastases of colorectal carcinoma have significantly greater lipid content, expressed as Lipids/Cr ratio, compared to metastatic tumors of other origin. The authors suggested an optimal Lipids/Cr cut off value of 2 for the identification of the colorectal carcinoma [108]. It is obvious that ${ }^{1} \mathrm{H}-$ MRS may aid in the determination of cerebral metastases origin, nevertheless, further research is needed to determine the exact role of proton MR spectroscopy in the identification of the tissue type of metastatic brain tumors.

\subsection{Meningiomas}

Meningiomas are common intracranial tumors and are generally easily diagnosed by their characteristic radiological imaging appearance of solid mushroom imaging pattern, extracranial location, dura matter conjunction and sinus involvement. However, $15 \%$ of meningiomas exhibit rim like enhancement, a prominent cystic component, hemorrhage, or even metaplasia [109], mimicing gliomas or cerebral metastatic tumors. ${ }^{1} \mathrm{H}-\mathrm{MRS}$ has been proved useful in differentiating meningiomas with atypical radiologic pattern from other brain tumors [36].

Alanine at $1.47 \mathrm{ppm}$ has been considered as the characteristic metabolic marker of meningiomas which differentiates them from other brain tumors [36, 38]. Nevertheless, reported occurrence of Alanine varies among different studies [37, 16] as it can significantly overlap with lactate resonance due to J-coupling effect [37]. 
In the absence of Alanine, several investigators aimed to correlate other metabolites to meningioma presence. Studying the metabolic profile of different cerebral tumors using short TE ${ }^{1} \mathrm{H}-\mathrm{MRS}$, Howe et al. found that low levels of $\mathrm{mI}$ and $\mathrm{Cr}$ were characteristic for meningiomas relative to grade II astrocytomas, anaplastic astrocytomas and glioblastomas [22]. In the same study meningiomas revealed the highest $\mathrm{Cho} / \mathrm{Cr}$ ratio among the other brain tumors, on both short and long TE. Another reported specific finding for meningiomas, is the absence of the neuronal marker NAA. Instead of partial volume effects [3], the peak of NAA at meningioma spectra, may also represent other endogenous NAA compounds (NACs) such as N-acetylaspartylglutamate, $\mathrm{N}$-acetylneuraminic acid and $\mathrm{N}$ acetylgalactosamine [37].

A recent study by Kousi et al. revealed a distinct chemical compound, observed in all meningiomas recruited for that study, which may establish a rather specific marker in their differential diagnosis from high grade gliomas and metastases [6]. This chemical substance, resonated at $3.8 \mathrm{ppm}$ using short TE ${ }^{1} \mathrm{H}$-MRS (Figure 8 ) and according to the in vitro study of Tugnoli et al. it might receive contribution from phosphoethanolamine (PE) and other amino acids such as Leukine, ALanine, Glutamate, Glutamine, Glutathione, Lysine, Arginine and Serine [110].
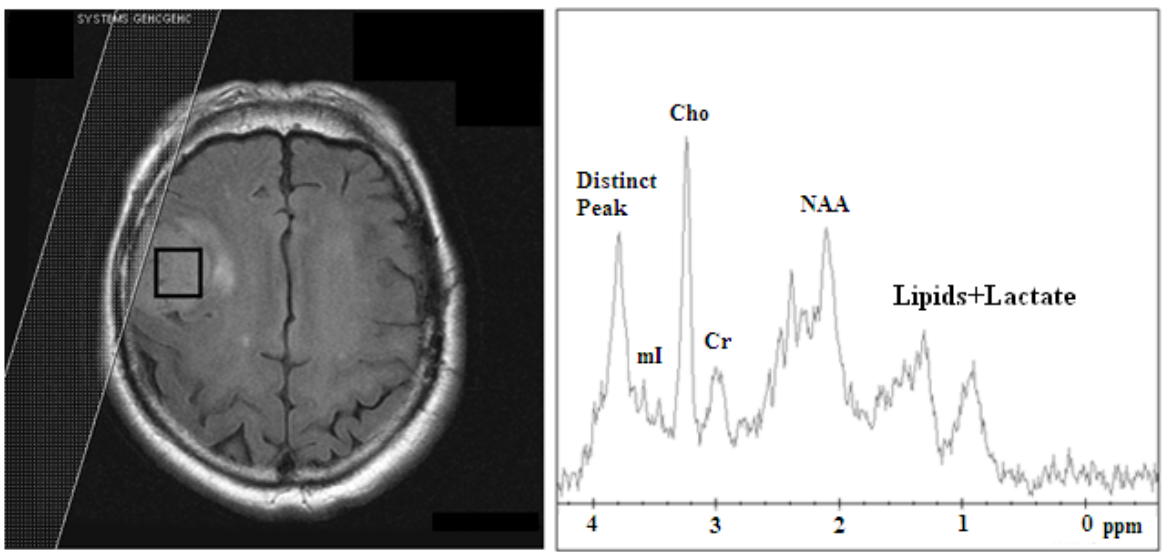

Figure 8. FLAIR T2 images (left) of a meningioma with its corresponding spectrum (right), at short TE (35ms). Elevated Cho and lipid resonances were detected at $3.2 \mathrm{ppm}$ and $1.3 \mathrm{ppm}$ respectively, as well as a distinct chemical compound resonating at $3.8 \mathrm{ppm}$ [6].

\subsection{Primary Central Nervous System Lymphomas (PCNSL)}

Primary central nervous system lymphoma (PCNSL) represents $1 \%$ of all brain tumors and its incidence has increased in the last 3 decades. Although densely contrast-enhancing lesions, without the presence of necrosis are characteristic imaging features of PCNS lymphoma, it can be difficult, sometimes even impossible, to distinguish PCNSLs from high grade gliomas on conventional MRI [111]. Their differentiation, however, has important diagnostic and therapeutic implications. 
For the correct diagnosis of brain lymphomas, ${ }^{1} \mathrm{H}-\mathrm{MRS}$ has reported promising results. The most specific finding for PCNSL on MRS is an increase in lipid and Cho resonances (Figure 9). Sometimes, lipid peaks in PCNSL may be more prominent than in high grade gliomas and can help differentiate between the two tumor types [107].

Lipids are typically a signature of cell death; however, a lipid dominated spectrum found in PCNSL does not indicate necrosis. This appears to be due to numerous macrophages and the increased turnover of membrane components in transformed lymphoid cells which contain high concentrations of mobile lipids [112].

Histopathologically, PCNSLs are characterized by a diffusely infiltrative pattern and hence, it is important to survey the peritumoral area also and not just the area of obvious tumor involvement. Like high grade gliomas, the peritumoral area of PCNSLs demonstrates an abnormal metabolite pattern. Chawla et al. reported increased $\mathrm{Cho} / \mathrm{Cr}$ and $\mathrm{Lip}+\mathrm{Lac} / \mathrm{Cr}$ ratios in the peritumoral area of PCNSLs. They also observed significantly higher Lip+Lac/Cr ratio in the peritumoral area of PCNSLs when compared with that of GBMs, suggesting the presence of infiltrative active lymphocytes and macrophages in areas beyond lymphoma boundaries. Using a threshold value of 7.09 for Lip+Lac/Cr ratio they differentiated PCNSLs from GBMs with $84.6 \%$ sensitivity and $75 \%$ specificity [107]. Therefore, in the absence of obvious necrosis, increased lipid concentration together with a markedly elevated $\mathrm{Cho} / \mathrm{Cr}$ ratio for both intratumoral and peritumoral areas can provide important metabolic information which may improve the distinction between PCNSLs and other brain tumors.
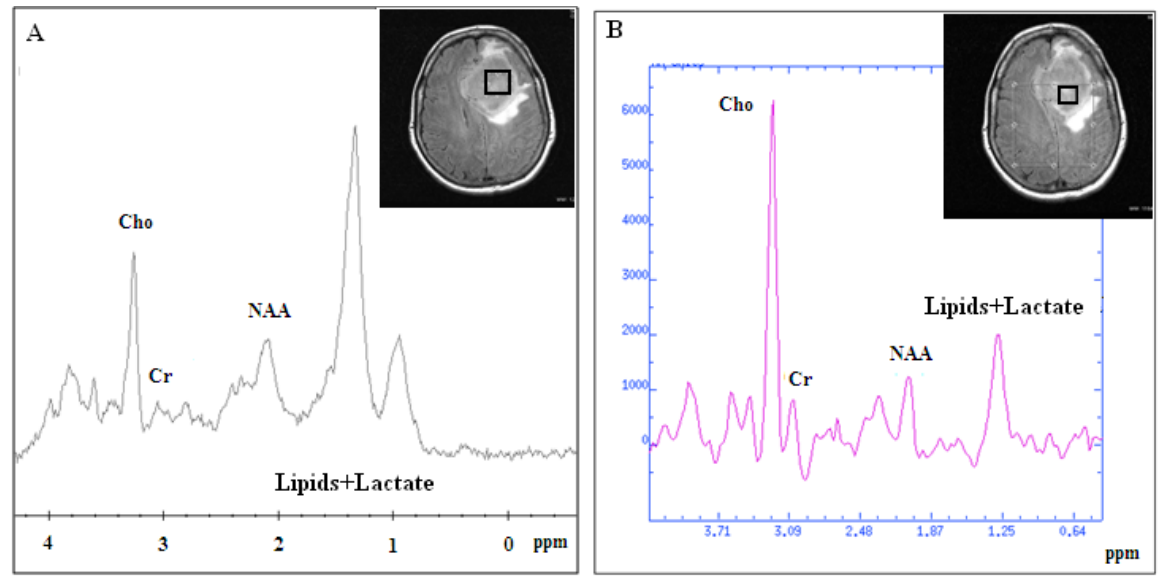

Figure 9. Spectra from an intracerebral lymphoma on both short (A) and long TE (B), demonstrating the characteristic elevation of lipid and Cho resonances.

\subsection{Central neurocytomas}

Central neurocytomas (CNCs) are a neuronal tumor almost exclusively located in the lateral ventricles that appear in young adults. Most of these tumors do not recur after surgery and are generally considered benign, with a favorable prognosis [28]. 
Instead of their typical radiological appearance - a well circumscribed lobulated mass in the anterior portion of the lateral ventricles- it may not always be possible to differentiate CNCs from other intraventricular tumors such as oligodendrogliomas and ependymomas [113].

${ }^{1} \mathrm{H}$-MRS has been proved a valuable tool for the presurgical diagnosis of these neoplasms. Previous studies have reported CNCs to consistently show the tumoral pattern of increased Cho and decreased NAA levels [28, 35]. On the contrary, lactate has not been observed consistently in all studies. Specifically, although Kim et al. reported lactate in all of their patients, Shah et al. observed lactate in only $9 \%$ of the CNC cases [114, 115]. A few studies have speculated the rather specific marker of Gly at 3.55ppm on long TE spectra, strongly suggesting CNC occurrence [28, 115].

The presence of Ala in a patient with CNC was first reported by Chuang et al. using a 3T MR system [116]. It was demonstrated as an inverted doublet at $1.5 \mathrm{ppm}$ with a TE of 135 msec. Similarly, Krishnamoorthy et al. also observed Ala in all three CNC cases $(100 \%)$ studied, whereas in the study of Shah et al. Ala was observed in $64 \%$ of the CNCs $[35,115]$.

Thus, CNCs may show Ala as an inverted doublet at $1.5 \mathrm{ppm}$ in long TE spectra. Although one may observe Ala in other intraventricular tumors such as meningioma, other characteristic peaks such as Gly, high Cho and decreased NAA should help to correclty identify CNC.

\subsection{Gliomatosis Cerebri}

Gliomatosis Cerebri (GC) is a rare brain tumor characterized by a diffuse neoplastic overgrowth of glial elements of various histological subtypes (astrocytoma, oligodendroglioma, or mixed glioma) and extensive infiltration of at least two lobes [117]. Unlike gliomas, the neuronal architecture is usually preserved [118].

MRI characteristics of GC are non-specific and occasionally it is difficult to differentiate GC from demyelinating diseases or viral encephalitis, and biopsy is often inconclusive [9]. Given the unfavorable prognosis of this tumor type, there is a demand for alternative imaging techniques, such as ${ }^{1} \mathrm{H}-\mathrm{MRS}$, to grade GC and to detect the most anaplastic areas for determining surgical areas and radiotherapeutic targets.

A few studies have looked at the spectral features of such tumors and those are consistent with the spectral features of gliomas discussed above. By studying 8 patients with GC using long TE ${ }^{1} \mathrm{H}-\mathrm{MRS}$, Bendszus et al. found elevated $\mathrm{Cho} / \mathrm{Cr}$ and $\mathrm{Cho} / \mathrm{NAA}$ ratios, as well as decreased NAA/Cr ratios of varying degrees in the abnormal areas on T2-weighted images [119]. Similarly, a retrospective analysis by $\mathrm{Yu}$ et al. also revealed high $\mathrm{Cho} / \mathrm{Cr}$ and Cho/NAA ratios and low NAA/Cr ratio within the areas of hyperintensity on T2-weighted images in 8 histopathologically confirmed patients with GC. Anaplastic areas had higher Cho/NAA ratio and the lactate doublet was present [117]. Apart from being beneficial in the grading of GC, ${ }^{1} \mathrm{H}$-MRS might reflect the true extent of neoplastic infiltration more accurately than MRI. Bendszus et al. found elevated Cho/Cr and Cho/NAA ratios in the 
tumor margins that appeared normal on T2- weighted images. Tumoral infiltration of the margin of the lesion that appeared normal on T2-weighted images was also confirmed by $\mathrm{Yu}$ et al. by observing increased Cho/NAA ratio in those areas [117].

From the aforementioned findings it can be concluded that ${ }^{1} \mathrm{H}-\mathrm{MRS}$ when combined to MRI findings may aid to GC diagnosis. Additionally, the determination of highly anaplastic areas and areas of tumoral infiltration may have a great impact in radiotherapy planning.

\subsection{Suprasellar tumors}

Pituitary adenomas and craniopharyngiomas, are the most frequent suprasellar space occupying lesions and are generally regarded as benign neoplasms of the pituitary gland. Nevertheless, with respect to the differential diagnosis of suprasellar masses, pituitary adenomas, craniopharyngiomas together with gliomas and meningiomas can be considered [120].

To date only a few cases of pituitary adenomas and craniopharyngiomas have been studied by in-vivo ${ }^{1} \mathrm{H}-\mathrm{MRS}[16,120,121]$, probably because of their relative rarity and the technical difficulties in obtaining in vivo high-quality spectra without artifacts is such a region [120]. In a study by Chernov et al., the vast majority of the 19 pituitary adenomas were characterized by a significant reduction of NAA peak, moderate elevation of Cho, and infrequent presence of small lipid and lactate peaks. This metabolic pattern differentiated them from low grade gliomas which showed a moderate decrease of NAA and Cr peaks. In the same study, craniopharyngiomas were typically characterized by a significant decrease of all metabolites and presence of multiple additional peaks which were possibly resulted from the presence of calcifications and microcysts within the investigated volume of tissue [16]. On the contrary, Sener et al. demonstrated very prominent peaks in the craniopharyngiomas between 0.5 and $1.5 \mathrm{ppm}$, which probably corresponded to lipid peaks. Histological findings also revealed high amounts of cholesterol, lipids and lactate in the cyst fluid correlating with their spectroscopic findings [120].

\section{Conclusion}

${ }^{1} \mathrm{H}$-MRS can provide important in vivo metabolic information, complementing morphological findings from conventional MRI in the clinical setting. This technique is an extremely valuable tool in solving difficult neurological cases and increase confidence in diagnosis; however, it should be always considered a supplementary tool to the patients' clinical history, examination, and conventional MRI when reaching the final diagnosis.

The future would be to combine 1H-MRS with other advanced magnetic resonance techniques such as Diffusion/Diffusion Tensor Imaging and Perfusion-weighted Imaging, which will potentially prove to be useful in both clinical and research settings. Ultimately, these advanced tools may be used in a multiparametric, algorithmic fashion to characterize tissue biology and dramatically improve tumor differential diagnosis. 


\section{Author details}

Evanthia Kousi and Ioannis Tsougos

Medical Physics Department, School of Medicine, University of Thessaly, Larissa, Greece

Kapsalaki Eftychia

Radiology Department, School of Medicine, University of Thessaly, Larissa, Greece

\section{References}

[1] Nelson, S. J. Multivoxel magnetic resonance spectroscopy of brain tumors. Mol Cancer Ther 2003;2(5) 497-507.

[2] Soares, D. P. and M. Law. Magnetic resonance spectroscopy of the brain: review of metabolites and clinical applications. Clin Radiol 2009; 64(1) 12-21.

[3] Moller-Hartmann, W., S. Herminghaus, et al. Clinical application of proton magnetic resonance spectroscopy in the diagnosis of intracranial mass lesions. Neuroradiology 2002; 44(5) 371-81.

[4] Jonathan H. Gillard, Adam D. Waldman, Peter B. Barker. Clinical MR Neuroimaging: Diffusion, Perfusion and Spectroscopy. Cambridge University Press; 2004.

[5] Bonekamp, D., M. A. Jacobs, et al. Advancements in MR Imaging of the Prostate: From Diagnosis to Interventions. Radiographics 2011; 31(3) 677-703.

[6] Kousi E, Tsougos I, Vasiou K, et al. Magnetic Resonance Spectroscopy of the Breast at 3T: Pre- and Post-Contrast Evaluation for Breast Lesion Characterization. ScientificWorld Journal. 2012; 2012754380

[7] Lee, C. W., J. H. Lee, et al. Proton magnetic resonance spectroscopy of musculoskeletal lesions at $3 \mathrm{~T}$ with metabolite quantification. Clin Imaging 2010; 34(1) 47-52.

[8] Pinker, K., A. Stadlbauer, et al. Molecular imaging of cancer: MR spectroscopy and beyond. Eur J Radiol.2012;81(3) 566-77

[9] Sibtain, N. A., F. A. Howe, et al. The clinical value of proton magnetic resonance spectroscopy in adult brain tumours. Clin Radiol 2007; 62(2) 109-19.

[10] Doelken MT, Mennecke A, et al. (2009) Multi-voxel magnetic resonance spectroscopy of cerebral metabolites in healthy adults at 3 Tesla. Acad Radiol 2009; 16 (12) 1493-1501.

[11] Tavora DG, Nakayama M, et al. Leukoencephalopathy with brainstem and spinal cord involvement and high brain lactate: report of three Brazilian patients. Arq Neuropsiquiatr 2007;65 (2B) 506-511.

[12] Wattjes MP, Harzheim M, et al. High field MR imaging and 1H-MR spectroscopy in clinically isolated syndromes suggestive of multiple sclerosis: correlation between metabolic alterations and diagnostic MR imaging criteria. J Neurol 2008;255(1) 56-639.

[13] Rosen Y, Lenkinski RE. Recent advances in magnetic resonance neurospectroscopy. Neurotherapeutics. 2007;4(3) 330-45.

[14] Mader I, Rauer S, Gall P, Klose U. (1)H MR spectroscopy of inflammation, infection and ischemia of the brain. Eur J Radiol 2008; 67 (2) 250-257. 
[15] Lin A, Ross BD, Harris K, Wong W. Efficacy of proton magnetic resonance spectroscopy in neurological diagnosis and neurotherapeutic decision making. NeuroRx 2005; 2 (2) 197-214

[16] Chernov MF, Ochiai $\mathrm{T}$, et al. Role of proton magnetic resonance spectroscopy in preoperative evaluation of patients with mesial temporal lobe epilepsy. J Neurol Sci 2009; 285 (1-2):212-219.

[17] Wang S, Poptani H, et al. Amyotrophic lateral sclerosis: diffusion-tensor and chemical shift MR imaging at 3.0 T. Radiology 2006; 239 (3) 831-838.

[18] Lai PH, Weng HH, et al. In vivo differentiation of aerobic brain abscesses and necrotic glioblastomas multiforme using proton MR spectroscopic imaging. AJNR Am J Neuroradiol 2008; 29 (8) 1511-1518

[19] Hayashi T, Kumabe T, et al. Inflammatory demyelinating disease mimicking malignant glioma. J Nucl Med 2003; 44 (4) 565-569

[20] Mascalchi M, Brugnoli R, et al. Single-voxel long TE 1H-MR spectroscopy of the normal brainstem and cerebellum. J Magn Reson Imaging 2002; 16(5) 532-537.

[21] Robin A. de Graaf . In vivo nmr spectroscopy: Principles and Techniques.Wiley;2007

[22] Howe FA, Barton SJ, et al.: Metabolic profiles of human brain tumors using quantitative in vivo $1 \mathrm{H}$ magnetic resonance spectroscopy. Magn Reson Med 2003; 49(2) 223-232.

[23] Gideon P, Henriksen O, Sperling B, et al. Early time course of N-acetylaspartate, creatine and phosphocreatine, and compounds containing choline in the brain after acute stroke. A proton magnetic resonance spectroscopy study. Stroke 1992; 23(11) 1566-1572, 1992.

[24] van der Graaf M: In vivo magnetic resonance spectroscopy: basic methodology and clinical applications. Eur Biophys J 2010; 39(4) 527-40.

[25] Castillo M, Smith JK and Kwock L. Correlation of myo-inositol levels and grading of cerebral astrocytomas. AJNR Am J Neuroradiol 2000; 21(9) 1645-1649.

[26] Mishra AM, Gupta RK, Jaggi RS, et al. Role of diffusion-weighted imaging and in vivo proton magnetic resonance spectroscopy in the differential diagnosis of ring-enhancing intracranial cystic mass lesions. J Comput Assist Tomogr 2004; 28(4) 540-547.

[27] Kizu O, Yamada K, Nishimura T. Proton chemical shift imaging in normal pressure hydrocephalus. AJNR Am J Neuroradiol 2001;22(9) 1659-64.

[28] Yeh IB, Xu M, Ng WH, et al. Central neurocytoma: typical magnetic resonance spectroscopy findings and atypical ventricular dissemination. Magn Reson Imaging 2008; 26(1) 59-64.

[29] Cecil KM. MR spectroscopy of metabolic disorders. Neuroimaging Clin N Am 2006; 16(1) 87-116

[30] Srinivasan R, Cunningham C, Chen A, et al. TE-averaged two-dimensional proton spectroscopic imaging of glutamate at 3 T. Neuroimage 2006;30(4) 1171-8.

[31] Simister RJ, McLean MA, Barker GJ and Duncan JS: Proton MR spectroscopy of metabolite concentrations in temporal lobe epilepsy and effect of temporal lobe resection. Epilepsy Res 2009; 83(2-3): 168-176. 
[32] Kantarci K, Reynolds G, Petersen RC, et al. Proton MR spectroscopy in mild cognitive impairment and Alzheimer disease: comparison of 1.5 and 3 T. AJNR Am J Neuroradiol 2003; 24(5) 843-849.

[33] Ricci R, Bacci A, Tugnoli V, et al. Metabolic findings on 3T 1H-MR spectroscopy in peritumoral brain edema. AJNR Am J Neuroradiol 2007; 28(7): 1287-1291.

[34] Malhotra HS, Jain KK, Agarwal A, et al. Characterization of tumefactive demyelinating lesions using MR imaging and in-vivo proton MR spectroscopy. Mult Scler 2009; 15(2) 193-203.

[35] Krishnamoorthy T, Radhakrishnan VV, Thomas B, et al. Alanine peak in central neurocytomas on proton MR spectroscopy. Neurora- diology 2007;49(7) 551-4.

[36] Majos C, Alonso J, Aguilera C, et al. Proton magnetic resonance spectroscopy ((1)H MRS) of human brain tumours: assessment of differences between tumour types and its applicability in brain tumour categorization. Eur Radiol 2003;13(3) 582-591.

[37] Yue Q, Isobe T, Shibata $Y$, et al. New observations concerning the interpretation of magnetic resonance spectroscopy of meningioma. Eur Radiol 2008; 18(12) 2901-2911.

[38] Lehnhardt FG, Bock C, Rohn G, Ernestus RI and Hoehn M. Metabolic differences between primary and recurrent human brain tumors: a $1 \mathrm{H}$ NMR spectroscopic investigation. NMR Biomed 2005; 18(6): 371-382.

[39] Kinoshita $Y$ and Yokota A. Absolute concentrations of metabolites in human brain tumors using in vitro proton magnetic resonance spectroscopy. NMR Biomed 1997;10(1) 2-12.

[40] Shirayama Y, Obata T, Matsuzawa D, et al.: Specific metabolites in the medial prefrontal cortex are associated with the neurocognitive deficits in schizophrenia: a preliminary study. Neuroimage 2010;49(3) 2783-2790.

[41] An L, Zhang Y, Thomasson DM, et al.: Measurement of glutathione in normal volunteers and stroke patients at $3 \mathrm{~T}$ using J-difference spectroscopy with minimized subtraction errors. J Magn Reson Imaging 2009; 30(2) 263-270.

[42] Opstad KS, Provencher SW, Bell BA, et al. Detection of elevated glutathione in meningiomas by quantitative in vivo 1H MRS. Magn Reson Med 2003;49(4) 632-637.

[43] Barker PB, Hearshen DO and Boska MD: Single-voxel proton MRS of the human brain at 1.5T and 3.0T. Magn Reson Med 2001;45(5) 765-769.

[44] Stephenson MC, Gunner F, Napolitano A, et al.: Applications of multi-nuclear magnetic resonance spectroscopy at 7T. World J Radiol 2011; 3(4) 105-113.

[45] Di Costanzo A, Trojsi F, Tosetti M, et al. High-field proton MRS of human brain. Eur J Radiol 2003; 48(2) 146-153.

[46] Lange T, Dydak U, Roberts TP, Rowley HA, Bjeljac M and Boesiger P: Pitfalls in lactate measurements at 3T. AJNR Am J Neuroradiol 2006;27(4) 895-901.

[47] Avdievich NI, Pan JW, Baehring JM, Spencer DD, Hetherington HP. Short echo spectroscopic imaging of the human brain at 7T using transceiver arrays. Magn Reson Med 2009;62(1)17-25.

[48] Drost DJ, Riddle WR and Clarke GD. Proton magnetic resonance spectroscopy in the brain: report of AAPM MR Task Group \#9. Med Phys 2002;29(9) 2177-2197. 
[49] Hetherington HP, Chu WJ, Gonen O, Pan JW. Robust fully automated shimming of the human brain for high-field $1 \mathrm{H}$ spectroscopic imaging. Magn Reson Med 2006;56(1)2633.

[50] Zhang Y, Li S, Shen J. Automatic high-order shimming using parallel columns mapping (PACMAP). Magn Reson Med 2009;62(4)1073-1079.

[51] Donald W. McRobbie, Elizabeth A. Moore, Martin J. Graves, Martin R. Prince From Image to Proton. Cambridge University Press; 2007

[52] Frahm J, Bruhn H, Gyngell ML, et al. Localized high-resolution proton NMR spectroscopy using stimulated echoes: initial applications to human brain in vivo. Magn Reson Med 1989;9(1) 79-93.

[53] Bottomley P. 1984. In U.S. Patent, Vol. 4480228 USA.

[54] Duyn JH, Gillen J, Sobering G, van Zijl PCM, Moonen CTW. Multislice proton MR spectroscopic imaging of the brain. Radiology 1993; 188(1) 277- 282.

[55] Gruber S, Mlynarik V and Moser E. High-resolution 3D proton spectroscopic imaging of the human brain at $3 \mathrm{~T}$ : SNR issues and application for anatomy-matched voxel sizes. Magn Reson Med 2003;49(2) 299-306.

[56] Haase A, Frahm J, Hanicke W, Matthei D. 1H NMR chemical shift selective imaging. 1985; Phys Med Biol 30(4) 341-344.

[57] Mescher M, Tannus A, O'Neil Johnson M, Garwood M. Solvent suppression using selective echo dephasing. J Magn Reson A 1996; 123, 226-229.

[58] Patt SL, Sykes BD. Water eliminated Fourier transform NMR spectroscopy. J Chem Phys 1972; 56, 3182-3184.

[59] Spielman DM, Pauly JM, Macovski A, Glover GH, Enzmann DR.. Lipid-suppressed single- and multisection proton spectroscopic imaging of the human brain. J Magn Reson Imaging 1992; 2(3): 253-262.

[60] Jissendi Tchofo $\mathrm{P}$ and Baleriaux D: Brain (1)H-MR spectroscopy in clinical neuroimaging at 3T. J Neuroradiol, 2008; 36(1) 24-40

[61] Coron A,Vanhamme L, Antoine JP,Van Hecke P,Van Huffel S. The filtering approach to solvent peak suppression in MRS: a critical review. J Magn Reson 2001;152(1) 26-40.

[62] Jiru F. Introduction to post-processing techniques. Eur J Radiol 2008;67(2) 202-217, 2008.

[63] Bakshi R, Thompson AJ, Rocca MA, et al. MRI in multiple sclerosis: current status and future prospects. Lancet Neurol 2008;7 (7) 615-625.

[64] De Stefano N and Filippi M. MR spectroscopy in multiple sclerosis. J Neuroimaging 2007; 17 Suppl 1: 31S-35S.

[65] Rovira A, Leon A. MR in the diagnosis and monitoring of multiple sclerosis: an overview. Eur J Radiol 2008; 67 (3):409-414.

[66] Butteriss DJ, Ismail A, Ellison DW, Birchall D.Use of serial proton magnetic resonance spectroscopy to differentiate low grade glioma from tumefactive plaque in a patient with multiple sclerosis. Br J Radiol 2003; 76 (909) 662-665

[67] Fernando KT, McLean MA, Chard DT, et al. Elevated white matter myo-inositol in clinically isolated syndromes suggestive of multiple sclerosis. Brain 2004;127(6) 13611369. 
[68] Kirov I, Patil V, Babb J, et al. MR Spectroscopy Indicates Diffuse Multiple Sclerosis Activity During Remission. J Neurol Neurosurg Psychiatry 2009; 80(12) 1330-6.

[69] Kimura T, Sako K, Gotoh T, et al. In vivo single-voxel proton MR spectroscopy in brain lesions with ring-like enhancement. NMR Biomed 2001; 14 (6) 339-349.

[70] Lai PH, Ho JT, Chen WL, et al. Brain abscess and necrotic brain tumor: discrimination with proton MR spectroscopy and diffusion weight imaging. Am J Neuroradiol 2002;23(8)1369-77.

[71] Kapsalaki EZ, Gotsis ED, Fountas KN (2008) The role of proton magnetic resonance spectroscopy in the diagnosis and categorization of cerebral abscesses. Neurosurg Focus 2008; 24 (6) E7.

[72] Kadota O, Kohno K, Ohue S, et al: Discrimination of brain abscess and cystic tumor by in vivo proton magnetic resonance spectroscopy. Neuro Med Chir (Tokyo) 2001;41(3) 121-126.

[73] Lai PH, Li KT, Hsu SS, et al: Pyogenic brain abscess: findings from in vivo 1.5-t and 11.7-t in vitro proton MR spectroscopy. AJNR Am J Neuroradiol 2005; 26(2) 279- 288.

[74] Saunders DE. MR spectroscopy in stroke. Br Med Bull 2000; 56 (2):334-345

[75] Graham GD, Blamire AM, Howseman AM, et al. Proton magnetic resonance spectroscopy of cerebral lactate and other metabolites in stroke patients. Stroke 1992; 23(3) 333-340.

[76] Munoz Maniega S, Cvoro V, Armitage PA, et al. Choline and creatine are not reliable denominators for calculating metabolite ratios in acute ischemic stroke. Stroke 2008; 39 (9) 2467-2469.

[77] Rumpel H, Lim WE, Chang HM, et al. Is myo-inositol a measure of glial swelling after stroke? A magnetic resonance study. J Magn Reson Imaging 2003; 17 (1) 11-19.

[78] Glodzik-Sobanska L, Slowik A, Kozub J, et al. GABA in ischemic stroke. Proton magnetic resonance study. Med Sci Monit 2004; 10 Suppl 3:88-93.

[79] Hajek M, Dezortova M, Krsek P. (1)H MR spectroscopy in epilepsy. Eur J Radiol 2008; 67 (2):258-267.

[80] Leite RA, Otaduy MC, Silva GE, et al. Diagnostic methods for extra-temporal neocortical focal epilepsies: present and future. Arq Neuropsiquiatr 2010; 68 (1) 119-126.

[81] Moffett JR, Ross B, Arun P, Madhavarao CN, Namboodiri AMA. N-Acetylaspartate in the CNS: from neurodiagnostics to neurobiology. Prog Neurobiol 2007;81(2) 89-131.

[82] Wellard RM, Briellmann RS, Prichard JW, et al. Myoinositol abnormalities in temporal lobe epilepsy. Epilepsia 2003; 44(6) 815-821.

[83] Doelken MT, Stefan H, Pauli E, et al. (1)H-MRS profile in MRI positive- versus MRI negative patients with temporal lobe epilepsy. Seizure 2008; 17 (6):490-497.

[84] Mueller, S. G., Schuff, N., and Weiner, M. W. Evaluation of treatment effects in Alzheimer's and other neurodegenerative diseases by MRI and MRS. NMR. Biomed. 2006; 19(6), 655-668.

[85] Chantal, S., Braun, C. M., Bouchard, R. W., et al. Similar $1 \mathrm{H}$ magnetic resonance spectroscopic metabolic pattern in the medial temporal lobes of patients with mild cognitive impairment and Alzheimer disease. Brain Res. 2004; 1003(1-2), 26-35. 
[86] Herminghaus, S., Frolich, L., Gorriz, C., et al. Brain metabolism in Alzheimer disease and vascular dementia assessed by in vivo proton magnetic resonance spectroscopy. Psychiatry. Res. 2003; 123(3), 183-190.

[87] Kantarci K, Jack CR, Xu YC, et al. Regional metabolic patterns in mild cognitive impairment and Alzheimer's disease: a 1H MRS study. Neurology 2000;55(2) 210-217

[88] Hattori, N., Abe, K., Sakoda, S., and Sawada, T. Proton MR spectroscopic study at 3 Tesla on glutamate/glutamine in Alzheimer's disease. Neuroreport 2002; 13(1), 183-186.

[89] Clarke, C.E. \& M. Lowry. 2001. Systematic review of proton magnetic resonance spectroscopy of the striatum in parkinsonian syndromes. Eur. J. Neurol. 2001; 8(6) 573577.

[90] Rango, M. Arighi A, Bonifati C, Bresolin N.Magnetic resonance spectroscopy in Parkinson's disease and parkinsonian syndromes. Funct. Neurol. 2007; 22(2) 75-79.

[91] Hu MT, Taylor-Robinson SD, Chaudhuri KR, et al. Evidence for cortical dysfunction in clinically non-demented patients with Parkinson's disease: a proton MR spectroscopy study. J Neurol Neurosurg Psychiatry 1999;67(1) 20-26.

[92] Choe BY, Park JW, Lee KS, et al. Neuronal laterality in Parkinson's disease with unilateral symptom by in vivo $1 \mathrm{H}$ magnetic resonance spectroscopy. Invest Radial 1998; 33(8) 450-455.

[93] Taylor-Robinson SD, Turjanski N, Bhattacharya S, et al. A proton magnetic resonance spectroscopy study of the striatum and cerebral cortex in Parkinson's disease. Metab Brain Dis 1999;14(1) 45-55.

[94] Griffith HR, den Hollander JA, Okonkwo OC et al. Brain N-acetylaspartate is reduced in Parkinson disease with dementia. Alzheimer. Dis. Assoc. Disord. 2008; 22(1) 54-60.

[95] Summerfield, C. et al. 2002. Dementia in Parkinson disease: a proton magnetic resonance spectroscopy study. Arch. Neurol.2002; 59(9) 1415-1420.

[96] Yuh EL, Barkovich AJ, Gupta N. Imaging of ependymomas: MRI and CT. Childs Nerv Syst 2009; 25(10) 1203-13.

[97] Rijpkema, M., J. Schuuring, et al. Characterization of oligodendrogliomas using short echo time 1H MR spectroscopic imaging. NMR Biomed 2003;16(1): 12-8.

[98] Majos, C., C. Aguilera, et al. In vivo proton magnetic resonance spectroscopy of intraventricular tumours of the brain." Eur Radiol 2009;19(8): 2049-59.

[99] Porto, L., M. Kieslich, et al. MR spectroscopy differentiation between high and low grade astrocytomas: a comparison between paediatric and adult tumours. Eur J Paediatr Neurol 2011; 15(3) 214-21.

[100] Law, M., S. Yang, et al. Glioma grading: sensitivity, specificity, and predictive values of perfusion MR imaging and proton MR spectroscopic imaging compared with conventional MR imaging. AJNR Am J Neuroradiol 2003; 24(10) 1989-98.

[101] Server, A., B. Kulle, et al. Measurements of diagnostic examination performance using quantitative apparent diffusion coefficient and proton MR spectroscopic imaging in the preoperative evaluation of tumor grade in cerebral gliomas." Eur J Radiol. 2011 Nov;80(2) 462-70. 
[102] Server, A., R. Josefsen, et al. Proton magnetic resonance spectroscopy in the distinction of high-grade cerebral gliomas from single metastatic brain tumors." Acta Radiol 2010; 51(3) 316-25.

[103] Cha, S. Neuroimaging in neuro-oncology. Neurotherapeutics 2009; 6(3): 465-77.

[104] Chawla, S., Y. Zhang, et al. Proton magnetic resonance spectroscopy in differentiating glioblastomas from primary cerebral lymphomas and brain metastases. J Comput Assist Tomogr 2010; 34(6) 836-41.

[105] Law, M., S. Cha, et al. High-grade gliomas and solitary metastases: differentiation by using perfusion and proton spectroscopic MR imaging. Radiology 2002;222(3) 715-21.

[106] Opstad, K. S., M. M. Murphy, et al. Differentiation of metastases from high-grade gliomas using short echo time 1H spectroscopy. J Magn Reson Imaging 2004; 20(2) 18792.

[107] Sjobakk, T. E., R. Johansen, et al. Metabolic profiling of human brain metastases using in vivo proton MR spectroscopy at 3T. BMC Cancer 2007; 7: 141.

[108] Chernov, M. F., Y. Ono, et al. Comparison of (1)H-MRS-detected metabolic characteristics in single metastatic brain tumors of different origin. Brain Tumor Pathol 2006; 23(1): 35-40.

[109] Hakyemez B, Yildirim N, Erdogan C, et al. Meningiomas with conventional MRI findings resembling intraaxial tumors: can perfusion-weighted MRI be helpful in differentiation? Neuroradiology2006; 48(10) 695-702.

[110] Tugnoli V, Schenetti L, Mucci A, et al. Ex vivo HR-MAS MRS of human meningiomas: a comparison with in vivo 1H MR spectra. Int J Mol Med. 2006;18(5) 859-69.

[111] Weber, M. A., S. Zoubaa, et al. Diagnostic performance of spectroscopic and perfusion MRI for distinction of brain tumors. Neurology 2006; 66(12): 1899-906.

[112] Tang, Y.Z., Booth, T.C., Bhogal, P. et al. Imaging of primary central nervous system lymphoma. Clin Radiol 2011; 66(8) 768-777.

[113] Koeller KK, Sandberg GD. Cerebral intraventricular neoplasms: radiologic-pathologic correlation. Radiographics 2002;22:1473-505.

[114] Kim DG, Choe WJ, Chang KH, et al. In vivo proton magnetic resonance spectroscopy of central neurocytomas. Neurosurgery 2000; 46 (2) 329-333

[115] Shah T, Jayasundar R, Singh VP, Sarkar C. MRS characterization of central neurocytomas using glycine. NMR Biomed 2011; 24 (10) 1408-1413.

[116] Chuang MT, Lin WC, Tsai HY, et al. 3-T proton magnetic resonance spectroscopy of central neurocytoma: 3 case reports and review of the literature. J Comput Assist Tomogr 2005; 29 (5) 683-688.

[117] $\mathrm{Yu} \mathrm{A}$, Li K, Li H. Value of diagnosis and differential diagnosis of MRI and MR spectroscopy in gliomatosis cerebri. Eur J Radiol 2006; 59 (2) 216-221.

[118] Arai M, Kashihara K, Kaizaki Y, et al. Gliomatosis cerebri: report of 3 cases and review of recent literatures. No To Shinkei 2003;55(10) 890-7.

[119] Bendszus M, Warmuth-Metz M, Klein R, et al. MR spectroscopy in gliomatosis cerebri. AJNR Am J Neuroradiol 2000; 21 (2) 375-380

[120] Sener RN. Proton MR spectroscopy of craniopharyngiomas. Comput Med Imaging Graph 2001; 25 (5):417-422. 
50 Novel Frontiers of Advanced Neuroimaging

[121] Stadlbauer A, Buchfelder M, Nimsky C, Saeger W, Salomonowitz E, Pinker K, Richter G, Akutsu H, Ganslandt $\mathrm{O}$ et al. Proton magnetic resonance spectroscopy in pituitary macroadenomas: preliminary results. J Neurosurg 2008; 109 (2) 306-312. 